\section{Mass Spectrometry for the Characterization of Microorganisms ACS Symposium Series 541}

Catherine Fenselau, Editor

American Chemical Society,

Washington, DC 20036

$1994, \$ 64.95$

Book review by Jaap J. Boon

FOM Institute for Atomic and Molecular Physics

Kruislaan 407

1098SJ Amsterdam, Netherlands

Microorganisms display a wide range of compounds (also known as biomarkers) that can be exploited for classification and rapid recognition. This book contains proceedings of the American Chemical Society Symposium on mass spectrometry for the characterization of microorganisms (204th meeting of the ACS, Washington, 1992). Fourteen contributions on how mass spectrometry is used to tap this source of information are included. Lipids (Chapters 2, 3, 4, and 14), carbohydrates (Chapters 8, 9, and 13), and $t$-RNA nucleosides (Chapter 10) are shown to have a multitude of structural differences that make these compounds useful for phylogenetic differentiation, identification of pathogens, and environmental studies.

Mass spectrometry is utilized in all its available forms - both as a direct probe monitor and hooked up to chromatographic inlets. Some of the compound classes, for example, carbohydrates, require considerable workup and pre-separation to be useful as marker compounds. The chapter by Fox and Black (Chapter 8) gives many details on the necessary derivatization reactions and shows that the approach is very rewarding. The $t$-RNA nucleosides (Chapter 10 by Edmonds et al.) are digested by nucleases on the nanomole level and directly analyzed with high performance liquid chromatography thermospray mass spectrometry. The extensive efforts of the McCloskey group have resulted in the structural characterization of many nucleosides by mass spectrometry and thus have made these markers an operational tool for phylogeny.

Other compounds such as phospholipids and related complex lipids can be monitored with fast-atom bombardment (FAB) mass spectrometry and FAB tandem mass spectrometry as mixtures after extraction or even directly from a bacterial smear. These more sophisticated mass spectrometric approaches reduce the need for a high degree of pre-separation, but few laboratories have these resources available on a routine basis. There are also chapters on more classical structural studies of lipid A, lipooligosaccharides from

(C) 1995 American Society for Mass Spectrometry $1044-0305 / 95 / \$ 9.50$

SSDI 1044-0305(95)00525-I
Neisserin and Haemophilus, and glycolipids from $\mathrm{My}$ colncterin that use state-of-the-art mass spectrometry. Lipid signatures of microorganisms in environmental matrices are reviewed by White and co-workers (Chapter 2).

The symposium organizers have taken the opportunity to present work on rapid microscale techniques such as field site, ultra short column pyrolysis gas chromatography mass spectrometry to characterize bacterial matter in complex matrices directly sampled in the field. Pyrolysis is a useful preparation technique to release marker compounds and certainly not as bad as Fenselau suggests in her editorial review of the book (page 5), provided that the mass spectrometry is done properly. In-source pyrolysis mass spectrometry under positive and negative chemical ionization conditions at FOM has shown that large molecules can be released if the internal energy of the pyrolysis products is minimized.

"Sniffing and tasting" techniques such as head space analysis of volatile metabolites (Chapter 6 by Emokhonov et al.) and membrane probe technology for liquid phase metabolites (Chapter 7 by Lauritzen and Lloyd) are presented as promising approaches when much information is known about the target organisms. These techniques indirectly identify the microorganisms much like doctors of old could identify diseases by the smell of their patients. The aim is, of course, the rapid recognition of the "bugs," which works only when unique metabolites are present. These techniques are very useful to monitor bioreactors, but also can be used to monitor gases or pharmaceuticals in the bloodstream after insertion of a miniature membrane inlet mass spectrometry probe in an artery or vein.

Very little information was presented about proteins or nucleic acids as potential biomarker molecules, which are the "goodies" still to come in the future when matrix-assisted laser desorption ionization and electrospray ionization mass spectrometry will be developed to identify biomarkers from microorganisms or their digests. The nice mass spectrometry work by Fenselau's group, which presents work on mapping the Gag protein in the HIV virus (Chapter 11), is a preview.

This book is recommended as a good start for anyone interested in an orientational course on how mass spectrometry can be applied to analyze complex biomaterials. The symposium is somewhat biased toward medical microbiology, but the methodology presented is certainly important reading for geochemists, marine chemists, environmental and medical microbiologists, and chemical taxonomists. It should be an eye opener for those who were taught that mass spectrometry is to be used only for molecular weight analysis of pure synthetic compounds. 